University of Warwick institutional repository: http://go.warwick.ac.uk/wrap This paper is made available online in accordance with publisher policies. Please scroll down to view the document itself. Please refer to the repository record for this item and our policy information available from the repository home page for further information.

To see the final version of this paper please visit the publisher's website. Access to the published version may require a subscription.

Author(s): ANTHONY MANNING

Article Title: The volume entropy of a surface decreases along the Ricci flow

Year of publication: 2004

Link to published

version: http://dx.doi.org/10.1017/S0143385703000415

Publisher statement: None 


\title{
The volume entropy of a surface decreases along the Ricci flow
}

\author{
ANTHONY MANNING \\ Mathematics Institute, University of Warwick, Coventry CV4 7AL, UK \\ (e-mail:akm@maths.warwick.ac.uk)
}

(Received 5 June 2003 and accepted in revised form 23 June 2003)

Abstract. The volume entropy, $h(g)$, of a compact Riemannian manifold $(M, g)$ measures the growth rate of the volume of a ball of radius $R$ in its universal cover. Under the Ricci flow, $g$ evolves along a certain path $\left(g_{t}, t \geq 0\right)$ that improves its curvature properties. For a compact surface of variable negative curvature we use a Katok-Knieper-Weiss formula to show that $h\left(g_{t}\right)$ is strictly decreasing.

1. Introduction and statement of results

The volume of a ball of radius $R$ in the universal cover $(\tilde{M}, \tilde{g})$ of a Riemannian manifold $(M, g)$ grows exponentially at a rate

$$
h(g):=\lim _{R \rightarrow \infty} R^{-1} \log \operatorname{vol}(B(x, R, g)) \geq 0
$$

called the volume entropy of $(M, g)[25]$; see also $[\mathbf{8}, \mathbf{2 8}]$. The geodesic flow on the unit sphere bundle of a fixed Riemannian manifold $(M, g)$ of negative sectional curvature has the Anosov or hyperbolic property and is a major example of a structurally stable flow, see [2] and [17, §17.6]. It is known that, in the case of non-positive sectional curvature [25] or indeed of no conjugate points [9], the topological entropy of the geodesic flow for $(M, g)$ is equal to the volume entropy $h(g)$. Various estimates have been found for this topological entropy and for $h_{\lambda}(g)$, the entropy of the geodesic flow with respect to the Liouville measure $\lambda$, see $[9,15,16,27,30,35]$.

Katok, Knieper and Weiss showed in [18, Theorem 3] that, for any $C^{1}$ path $t \mapsto g_{t}$ of $C^{2}$ Riemannian metrics of negative sectional curvature on $M$, the entropy $h\left(g_{t}\right)$ depends $C^{1}$ on $t$ and has a derivative at $t=0$ given by

$$
h^{\prime}\left(g_{0}\right) / h\left(g_{0}\right)=-\left.\frac{1}{2} \int_{S M_{g_{0}}}(\partial / \partial t)\right|_{t=0} g_{t}(v, v) d \mu_{0}(v),
$$

where $\mu_{0}$ denotes the measure of maximal entropy for the geodesic flow for $\left(M, g_{0}\right)$ on its unit tangent bundle $S M_{g_{0}}$. 
The Ricci flow was introduced by Hamilton in [10]; see [6] for a recent survey. Starting from $\left(M, g_{0}\right)$ this flow provides a path $t \mapsto g_{t}(t \geq 0)$ of Riemannian metrics satisfying the partial differential equation

$$
\frac{\partial}{\partial t} g_{i j}=-2 R_{i j}+\frac{2}{n} r g_{i j}
$$

where $R_{i j}$ denotes the Ricci tensor, $n$ is the dimension of $M$ and $r$ denotes the average scalar curvature. The term $(2 / n) r g_{i j}$ ensures that the volume of $\left(M, g_{t}\right)$ is constant. In the case $n=2$ that interests us, equation (2) reduces to

$$
\frac{\partial}{\partial t} g_{i j}=-2(K-\bar{K}) g_{i j}
$$

where $K$ denotes the Gaussian curvature at a point of $M$ and $\bar{K}$ is its average value.

Standing hypothesis 1. From now on we assume that $M$ is a smooth closed surface of negative Euler characteristic $\chi(M)$.

Any Riemannian metric $g_{0}$ on $M$ of area 1 is conformally equivalent to a metric, $g_{\infty}$ say, of constant curvature and area 1. By Gauss-Bonnet, the value of this constant curvature is $2 \pi \chi(M)<0$ and then

$$
h\left(g_{\infty}\right)=\sqrt{-2 \pi \chi(M)},
$$

the growth rate of any unstable Jacobi field. All metrics in the flow $t \mapsto g_{t}$ are in the same conformal equivalence class, that is $g_{t}=e^{2 u_{t}} g_{\infty}$ for functions $u_{t}: M \rightarrow \mathbb{R}$ with $\int_{M} e^{2 u_{t}} d A_{\infty}=1$, where $d A_{\infty}$ denotes the area form on $\left(M, g_{\infty}\right)$. Katok showed that

$$
h\left(g_{0}\right) / h\left(g_{\infty}\right) \geq 1 / \int_{M} e^{u_{0}} d A_{\infty}>1,
$$

unless $\left(M, g_{0}\right)$ itself has constant curvature; see [15], and note that the argument in [16, p. 141] applies to $h\left(g_{0}\right)$ and not only to the topological entropy of the geodesic flow on $\left(M, g_{0}\right)$.

In [5], Besse discussed trends in Riemannian geometry including entropy and the Ricci flow (but not the relation between these). In [11, Theorem 3.3], Hamilton showed that, for any initial Riemannian metric $g_{0}$ on a surface, the Ricci flow converges to the metric $g_{\infty}$ of constant curvature. Moreover, if $\left(M, g_{0}\right)$ has negative curvature then so does $\left(M, g_{t}\right)$ for $t>0$ and the convergence is exponential. The Ricci flow has the differentiability we need to apply (1) because, by [37, Theorem 6.1] and [22], if $g_{0}$ is $C^{2}$ (or only in $H^{2}$ ) then $u_{t}(x)$ is $C^{\infty}$ on $M \times(0, \infty)$.

THEOREM 1. Let $M$ denote a smooth closed surface of negative Euler characteristic. Let $g_{0}$ denote a Riemannian metric on $M$ of non-constant negative curvature and area 1 , and let $g_{t}, t \geq 0$ denote the Ricci flow starting from $g_{0}$. Then the volume entropy $h\left(g_{t}\right)$ is a strictly decreasing function of $t$.

Remark 1. $h\left(g_{t}\right)$ converges to the value $\sqrt{-2 \pi \chi(M)}$ for constant curvature exponentially fast, using Theorem 3.3 of [11] and Sinaú's estimate [36] that $h$ lies in $\left[(n-1) k_{2},(n-1) k_{1}\right]$ when $n=\operatorname{dim} M$ and all sectional curvatures lie in $\left[-k_{1}^{2},-k_{2}^{2}\right]$. Volume entropy seems unrelated to the entropy $-\int_{M} K \log K$ shown by Hamilton to be increasing (provided $K>0$ ), see [11, Theorem 7.2]. 
Note that, under the Ricci flow, the Riemannian metric $g_{t}$ evolves to lengthen paths in regions of stronger than average negative curvature and to shorten them in regions of weaker than average curvature in order to bring the curvature towards a common value, while maintaining the area at a constant value. For fixed large $R$, the disc $B\left(x, R, g_{t}\right)$ evolves to reach further in the direction of geodesics passing through weaker than average negative curvature and less far in the direction of geodesics along which the curvature is more strongly negative than average, while the area of a fundamental domain is kept constant. The theorem states that the combined effect is always to reduce the growth rate. This accords with the intuition that, as $t$ increases, $B\left(x, R, g_{t}\right)$ gains translates of a fundamental domain along narrower strips than the broad neighbourhoods of geodesics experiencing stronger negative curvature where it relinquishes such translates.

If $\left(M, g_{0}\right)$ has conjugate points, then its geodesic flow does not satisfy the Anosov property $[3,19,24]$. In [33] Ruggiero shows that, in the space of $C^{2}$ Riemannian metrics on $M$ with the $C^{2}$ topology, the open set of metrics for which the geodesic flow on the unit tangent bundle satisfies the Anosov property is the interior of the closed set of those metrics that have no conjugate points.

THEOREM 2. Let $M$ denote a smooth closed surface of negative Euler characteristic and $g_{0} a C^{2}$ Riemannian metric. If the geodesic flow for $\left(M, g_{0}\right)$ is Anosov, then there is $T>0$ such that $h\left(g_{t}\right)$ is a strictly decreasing function of $t$ on $[0, T)$.

\section{Proofs and questions}

Proof of Theorem 1. An easy argument, using

$$
B(x, R(1-\varepsilon), \hat{g}) \subset B(x, R, g) \subset B(x, R(1+\varepsilon), \hat{g})
$$

if the ratio of the $g$-length to the $\hat{g}$-length of all vectors is between $1 \pm \varepsilon$, shows that $h\left(g_{t}\right)$ depends continuously on $t$. We choose $\tau>0$ and argue that $h^{\prime}\left(g_{\tau}\right)<0$. Because $h\left(g_{\tau}\right)>0$, it suffices from (1) to show that

$$
\left.\frac{1}{2} \int_{S M_{g_{\tau}}}(\partial / \partial t)\right|_{t=\tau} g_{t}(v, v) d \mu_{\tau}(v)>0
$$

By the definition of the Ricci flow (3), we need

$$
\int_{S M_{g_{\tau}}}-(K-\bar{K}) d \mu_{\tau}(v)>0
$$

or, equivalently,

$$
\int_{S M_{g \tau}}-K d \mu_{\tau}(v)>\int_{S M_{g \tau}}-\bar{K} d \mu_{\tau}(v)=-\bar{K}=-2 \pi \chi(M)>0 .
$$

This is a requirement that the measure of maximal entropy gives more weight to vectors in those parts of $M$ where the curvature is more strongly negative.

Now

$$
h\left(g_{\tau}\right)=h_{\mu_{\tau}} \leq \chi_{\mu_{\tau}},
$$


where $\mu_{\tau}$ is the measure of maximal entropy for the geodesic flow in $S M_{g_{\tau}}$ and $\chi_{\mu_{\tau}}$ is its positive Lyapunov exponent, namely the exponential growth rate of Jacobi fields along $\mu_{\tau}$-almost every geodesic, see $[\mathbf{3 2}, \mathbf{2 3}, \mathbf{4}]$. The inequality is due to Ruelle, see [34] and [23, §IV.12].

In [27, Theorem 1], we analysed the Riccati equation

$$
d a / d s=-a^{2}(s)-K(s)
$$

coming from the Jacobi equation along an orbit of the geodesic flow $\phi_{s}, s \in \mathbb{R}$ for $\left(M, g_{\tau}\right)$ that is typical for the Liouville measure $\lambda_{\tau}$. Let us apply this analysis to an orbit that is typical for the measure $\mu_{\tau}$ of maximal entropy. Then, for $\mu_{\tau}$ almost every $v \in S M_{g_{\tau}}$, we have

$$
\begin{aligned}
\chi_{\mu_{\tau}} & =\lim _{S \rightarrow \infty} S^{-1} \int_{0}^{S} a\left(\phi_{S}(v)\right) d s \\
& =\int_{S M_{g_{\tau}}} a d \mu_{\tau} \\
& \leq \sqrt{\int_{S M_{g \tau}} a^{2} d \mu_{\tau}} \\
& =\sqrt{\int_{S M_{g \tau}}-K d \mu_{\tau} .}
\end{aligned}
$$

This estimate (which is a special case of Corollary II.1 in [9]), together with (4), (5), (9), implies that $\int-K d \mu_{\tau}>-2 \pi \chi(M)$ as required for (8).

Proof of Theorem 2. Choose $T$ by structural stability $[2,17]$ so that the geodesic flow for $\left(M, g_{t}\right)$ is Anosov for $t \in[0, T)$. Expression (1) comes from Theorem 3 of [18], proved for negative curvature. But that Theorem 3 depends on the estimates in Proposition 8, whose proof also works for a small perturbation of an Anosov geodesic flow by comparing length in the two metrics for each almost uniformly distributed closed orbit. Thus (1) still holds in our case and the same argument goes through.

Question 1. Does the Ricci flow starting at a metric with geodesic flow of Anosov type stay within the class of such metrics or can conjugate points develop? Is the class of such metrics path connected?

Question 2. A surface $(M, g)$ of negative curvature can, through a large perturbation that glues a small pair of pants with two caps of positive curvature in place of a small disc, develop conjugate points and large topological entropy with only a small change to the volume entropy (to maintain area 1) [26]. Is the volume entropy strictly decreasing under the Ricci flow in the presence of conjugate points? One might restrict attention to the subspace of minimizing geodesics [29] and [7, Theorem 1.1].

Question 3. In $[\mathbf{1 5}, \mathbf{2 7}]$, the Liouville entropy $h_{\lambda}(g)$ is shown to be less than in the case of constant curvature. Is $h_{\lambda_{t}}\left(g_{t}\right)$ a strictly increasing function of $t$ ? (It is a $C^{1}$ function by [20].) By [11], $h_{\lambda_{t}}\left(g_{t}\right)$ certainly converges exponentially to the value $\sqrt{-2 \pi \chi(M)}$ using [27] or Pesin's formula [32] and [23, §IV.13]. 
Question 4. In [16, §4], Katok shows that the Cheeger isoperimetric constant $C_{g_{0}}$ (the infimum of the quotient of the length of a long simple closed curve $L$ in $\left(\tilde{M}, \tilde{g}_{0}\right)$ by the area it encloses) is at most $\int_{M} e^{u_{0}} d A_{\infty} \sqrt{-2 \pi \chi(M)}$ which is less than $C_{g_{\infty}}=$ $\sqrt{-2 \pi \chi(M)}$, the value in the case of constant curvature $2 \pi \chi(M)$; and he argues that $C_{g_{0}}$ is approached when $L:\left[0, \ell_{0}\right] \rightarrow \widetilde{M}$ is a closed curve of constant geodesic curvature. Is $C_{g_{t}}$ a strictly increasing function of $t$ ? (Hamilton shows in [13] that a certain isoperimetric constant on the 2-sphere improves along the Ricci flow.)

Question 5. Is there any result similar to Theorem 1 in dimension greater than 2 , say in a neighbourhood of a metric of constant curvature? It is not clear whether one should follow the Yamabe flow [21, 1], which converges to a metric of constant scalar curvature within the conformal class, or the Ricci flow, which is not currently known to converge to an Einstein metric of constant Ricci curvature, $[\mathbf{1 2}, \mathbf{1 4}]$; but see [31] (where the entropy seems unrelated to the volume entropy).

\section{REFERENCES}

[1] M. Anderson. Scalar curvature, metric degenerations and the static vacuum Einstein equations on 3-manifolds I. Geom. Funct. Anal. 9 (1999), 855-967.

[2] D. Anosov. Geodesic flows on closed Riemannian manifolds of negative curvature. Trudy Mat. Inst. Steklov 90 (1967) (in Russian) (English transl. Proc. Steklov Inst. 90).

[3] D. Anosov. Geodesic flows that satisfy the $U$-condition (Y). Trudy Mat. Inst. Steklov 167 (1985), 3-24 (in Russian).

[4] L. Barreira and Ya. Pesin. Lyapunov Exponents and Smooth Ergodic Theory (University Lecture Series, 23). American Mathematical Society, Providence, RI, 2002.

[5] A. Besse. Some trends in Riemannian geometry. Duration and Change. Springer, Berlin, 1994, pp. 71-105.

[6] H.-D. Cao and B. Chow. Recent developments on the Ricci flow. Bull. Amer. Math. Soc. 36 (1999), 59-74.

[7] C. Croke, A. Fathi and J. Feldman. The marked length-spectrum of a surface of nonpositive curvature. Topology 31 (1992), 847-855.

[8] E. Dinaburg. A connection between various entropy characterizations of dynamical systems. Izv. Akad. Nauk SSSR Ser. Mat. 35 (1971), 324-366 (in Russian).

[9] A. Freire and R. Mañé. On the entropy of the geodesic flow in manifolds without conjugate points. Invent. Math. 69 (1982), 375-392.

[10] R. Hamilton. Three-manifolds with positive Ricci curvature. J. Diff. Geom. 17 (1982), 255-306.

[11] R. Hamilton. The Ricci flow on surfaces. Mathematics and General Relativity (Contemporary Mathematics, 71). American Mathematical Society, Providence, RI, 1988, pp. 237-262.

[12] R. Hamilton. The formation of singularities in the Ricci flow. Surveys in Differential Geometry. Vol. II. International Press, Cambridge, MA, 1995, pp. 7-136.

[13] R. Hamilton. An isoperimetric estimate for the Ricci flow on the two-sphere. Modern Methods in Complex Analysis (Annals of Mathematics Studies, 137). Princeton University Press, Princeton, NJ, 1995, pp. 191200

[14] R. Hamilton. Non-singular solutions of the Ricci flow on three-manifolds. Comm. Anal. Geom. 7 (1999), 695-729.

[15] A. Katok. Entropy and closed geodesics. Ergod. Th. \& Dynam. Sys. 2 (1982), 339-365.

[16] A. Katok. Four applications of conformal equivalence to geometry and dynamics. Ergod. Th. \& Dynam. Sys. 8* (1988), 139-152.

[17] A. Katok and B. Hasselblatt. Introduction to the Modern Theory of Dynamical Systems. Cambridge University Press, Cambridge, 1995. 
[18] A. Katok, G. Knieper and H. Weiss. Formulas for the derivative and critical points of topological entropy for Anosov and geodesic flows. Comm. Math. Phys. 138 (1991), 19-31.

[19] W. Klingenberg. Riemannian manifolds with geodesic flow of Anosov type. Ann. Math. 99 (1974), 1-13.

[20] G. Knieper and H. Weiss. Regularity of measure theoretic entropy for geodesic flows of negative curvature I. Invent. Math. 95 (1989), 579-589.

[21] J. Lee and T. Parker. The Yamabe problem. Bull. Amer. Math. Soc. 17 (1987), 37-91.

[22] G. Lieberman. Second Order Parabolic Differential Equations. World Scientific, Singapore, 1996.

[23] R. Mañé. Ergodic Theory and Differentiable Dynamics. Springer, Berlin, 1987.

[24] R. Mañé. On a theorem of Klingenberg. Dynamical Systems and Bifurcation Theory (Pitman Research Notes Mathematical Series, 160). Longman, Harlow, 1987, pp. 319-345.

[25] A. Manning. Topological entropy for geodesic flows. Ann. Math. 110 (1979), 567-573.

[26] A. Manning. More topological entropy for geodesic flows. Dynamical Systems and Turbulence Warwick 1980 (Lecture Notes in Mathematics, 898). Springer, Berlin-New York, 1981, pp. 243-249.

[27] A. Manning. Curvature bounds for the entropy of the geodesic flow on a surface. J. London Math. Soc. 24 (1981), 351-357.

[28] J. Milnor. A note on curvature and fundamental group. J. Diff. Geometry 2 (1968), 1-7.

[29] M. Morse. A fundamental class of geodesics on any closed surface of genus greater than one. Trans. Amer. Math. Soc. 26 (1924), 25-60.

[30] R. Osserman and P. Sarnak. A new curvature invariant and entropy of geodesic flows. Invent. Math. 77 (1984), 455-462.

[31] G. Perelman. The entropy formula for the Ricci flow and its geometric applications. http://arxiv.org/abs/math.DG/0211159

[32] Ja. Pesin. Characteristic Ljapunov exponents and smooth ergodic theory. Russian Math. Surveys 32(4) (1977), 55-114.

[33] R. Ruggiero. On the creation of conjugate points. Math. Z. 208 (1991), 41-55.

[34] D. Ruelle. An inequality for the entropy of differentiable maps. Bol. Soc. Brasil. Mat. 9 (1978), 83-87.

[35] P. Sarnak. Entropy estimates for geodesic flows. Ergod. Th. \& Dynam. Sys. 2 (1982), 513-524.

[36] Ja. G. Sină. Asymptotic behavior of closed geodesics on compact manifolds with negative curvature. Izv. Akad. Nauk SSSR Ser. Mat. 30 (1966), 1275-1296 (in Russian).

[37] M. Struwe. Curvature flows on surfaces. Annali Sc. Norm. Sup. Pisa Serie V. 1 (2002), 247-274. 